\title{
PRÉSENTATION DE LA CARTE DES NAPPES D'EAU SOUTERRAINE DE LA FRANCE
}

$1 / 1000000^{\circ}$

\section{PAR J. MARGAT *}

Les problèmes d'eau de toute nature prennent, on le sait, une importance croissante et se posent de plus en plus à un niveau régional, alors qu'ils n'ont été longtemps considérés qu'à un niveau local. Les responsables et les cadres techniques des administrations centrales et régionales, comme ceux de nombreux organismes qui ont à connaître de ces problèmes, désirent disposer d'informations claires leur permettant à la fois d'orienter leurs décisions et de poser à bon escient des questions aux spécialistes.

Cependant, entre le niveau des travaux scientifiques publiés par ces spécialistes - ou encore trop souvent inédits et de diffusion limitée - - et celui des préoccupations pratiques des responsables des actions à entreprendre dans le domaine de l'eau, un écart subsiste fréquemment. Cet écart est d'ailleurs naturel et fondé, car les spécialistes savent qu'ils ne peuvent généralement pas fournir tout ce que les utilisateurs attendent d'eux, ni surtout aussi vite, et la simple probité scientifique les retient sur le chemin incertain des généralisations prématurées.

Le possible est à cet égard, comme à bien d'autres encore, bien en decà du souhaitable. Trop souvent, en France, l'étude des solutions qu'appellent des problèmes d'eau importants et urgents est retardée par l'absence des connaissances de base, dont l'acquisition exige non seulement des moyens, mais aussi des délais généralement non compressibles.

Néanmoins, l'écart en question peut et doit être au moins atténué, sinon comblé, et il est bon que, sous la pression des utilisateır's, les spécialistes tentent un effort de vulgarisation des commaissances, même limitées et imparfaites, sous forme de synthèse, certes provisoire, mais quelle synthèse ne lest pas?

Pour présenter clairement la répartition géographique des informations relatives à l'eau, il était

\footnotetext{
* Chef du Service d'Hydrogéologie du B.R.G.M.
}

normal de faire appel à la cartographie. Il sortirait du cadre de cette présentation d'aborder les nombreuses questions soulevées par la cartographie en Hydrologie, ou plus particulièrement en Hydrogéologie, et qui ont déjà été traitées. Rappelons seulement que tout n'est pas cartographiable en Hydrologie : une carte n'est que l'un des deux volets, nécessaire mais non suffisant, de la synthèse des connaissances, l'autre étant le bilan. Une carte représente des données spatiales relativement constantes. S'agissant d'une carte de vulgarisation, il est d'autant plus nécessaire de se tenir dans les limites de validité des données représentées.

C'est dans cet esprit, et dans le cadre de leurs attributions, que le Commissariat général du Plan d'équipement et de la Productivité, et le Secrétariat permanent pour l'étude des problèmes de l'eau de la Délégation a l'aménagement du Territoire et à l'action régionale, ont fait dresser par le B.R.G.M. une Carte des nappes d'eau souterraine de la France, sans méconnaître les difficultés ni les dangers qu'appelle toute cuvre de schématisation ni craindre les critiques - d'ailleurs opposées - qu'elle pourrait susciter.

Objet de la carte

Conformément à son titre, la carte se limite au domaine des eaux sonterraines. La figuration des principaux cours d'eau de surface n'a qu'un but topographique. La séparation établie entre les eaux souterraines et les eaux de surface ne signifie pas que les unes et les autres constituent des ressources en eau distinctes et indépendantes. Bien au contraire, on sait que les réserves d'eau souteraines fournissent la base et assurent la continuité des écoulements des cours d'eau de surface. Mais cette interdépendance est surtout à considérer dans le temps et l'objet de la carte était d'abord, on l'a dit, 


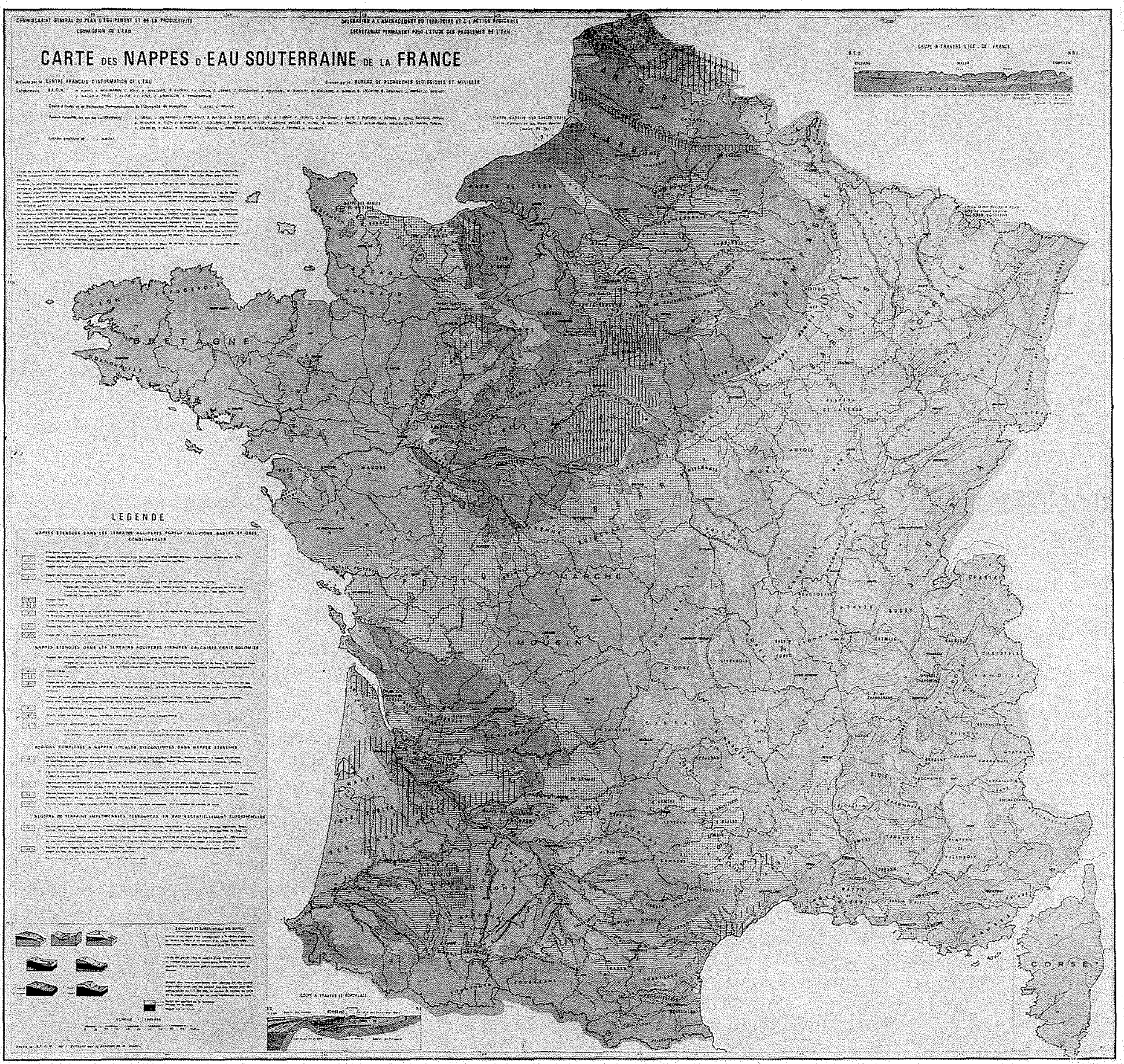

La réduction de la carte originale et sa reproduction en noir ne donnent qu'une idée sommaire de soll contenu. La légende comporte 15 classes réparties en 4 groupes :

1. Nappes étendues dans les terrains aquifères poreux : alluvions, sables, grès, conglomérats (nappes de vallées et de plateaux sableux, nappes captives des bassins de Paris et d'Aquitaine), en blen.

2. Nappes étendues dans les terrains aquifères fissurés: calcaires, craie, dolomies (réseaux aquifères des plateaux des grands bassins sédimentaires, et des massifs calcaires jurassiens, alpins et provencaux), en vert;

3. Régions complexes à nappes ou réseaux aquifères locaux et discontinus, sans nappes étendues formations semi-perméables, systèmes lenticulaires, régions plissées et volcaniques), en brun;

4. Régions de terrains imperméables, mais n'excluant pas l'existence de nappes profondes (parties argileuses et marneuses des bassins sédimentaires, massifs anciens), en brun. 228
This reduced black and white version of the original map only gives a sketchy idea of its contents. The full legend includes 15 classes divided into four main groups, i.e.

1. Extensive water tables in porous water-bearing soils such as allubia, sand, sandstone and conglomerates (examples; balley and sandy plateanx water tables and captive acquifers in the laris and Aquitaine basins). These are shown in blue;

2. Extensive water tables in fissured water-bearing forma tions such as limestone, chalk, and dolomite, e.g. groundwater networks of the plateaux of the great sedimentary basins and the Jurassic, Alpine or Provencal limestone mas sifs. These are shoun in green:

3. Complex regions with local or intermittent water tables or water-bearing systems, without extensive water tables, eg semi-penmeable formation lenticular systems and folded and volcanic regions. These are shown in brown: 4. Regions of impermeable ground but with the possibility of deep-lying water tables, e.g. clay or marl areas in sedimentarl basins and ancient formations. These are shown in brown. 
de représenter les données d'espace relativement stables.

Il s'agit ici d'une géographie simplifiée des eaux souterraines, comparable à la géographie des eaux de surface que sont les cartes hydrographiques classiques, et non d'une répartition des ressources en eau. L'objet de la carte est donc limité : il est de représenter schématiquement la situation et l'extension géographique des nappes d'eau souterraine les plus importantes, classées selon les grands types de terrains aquifères correspondants. En outre, plutôt que de laisser en blanc les régions dépourvues de nappes ou de réseaux aquifères étendus, on a préféré les subdiviser en un petit nombre de types, selon la fréquence relative et les types de nappes locales qui s'y trouvent :

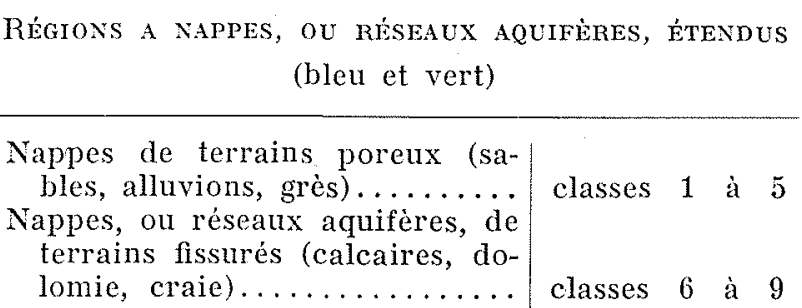

RÉgions dépouRvues de NaPpes Étendues (brun)

Régions complexes à alternance de terrains perméables et imperméables, à nappes locales discontinues. ............. classes 10 à 13

Régions de terrains imperméa-

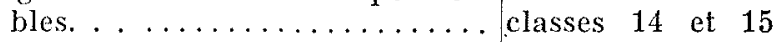

Ce classement concerne surtout les premières nappes rencontrées depuis le sol, ou «nappes phréatiques $\gg$. Mais dans les limites permises par la nécessité de ne pas nuire à la clarté de la carte, la représentation des principales nappes profondes captives a été aussi tentée autant que possible (classes $1^{\prime}, 3^{\prime}$ et $3^{\prime \prime}, 6^{\prime}$ et 9 ).

Ainsi conçue, la carte ne comporte volontairement aucune information quantitative sur les ressources en eau souterraine : ni sur la productivité des couches aquifères, ni sur l'alimentation naturelle des nappes, parce que les connaissances à ce sujet sont encore trop fragmentaires et souvent incertaines et aussi parce que, de toute manière, ces notions ne seraient pas intégralement ni valablement cartographiables, même à plus grande échelle.

Toutefois, la distinction majeure entre les régions à nappes d'eau souterraine étendues et celles qui en sont dépourvues est en même temps un partage du point de vue de l'importance relative des ressources en eau souterraine.

Dans les premières (classes 1 à 9 ), bien que très inégales selon les régions, les ressources en eau constituées par les nappes présentent une impor- tance régionale comparable à celle des eaux de surface. Leur conservation en vue d'une exploitation équilibrée et rationnelle et leur protection contre la pollution sont d'intérêt général.

Dans les secondes, les eaux souterraines, de par la nature du sous-sol, n'existent qu'en nappes discontinues et d'extension limitée (classes 10 à 15). Elles ne présentent alors qu'un intérêt local. Dans ces régions, les ressources en eau de surface, d'ailleurs souvent abondantes, sont seules capables de satisfaire les besoins en eau d'importance régionale.

L'ensemble constitue une première ébauche, assurément perfectible, de classification hydrogéologique régionale de la France. Basée sur une documentation à la fois très inégale selon les régions, selon les états d'avancement des connaissances, et incomplète à cause de l'extrême dispersion des données relatives aux eaux souterraines, cette carte manque inévitablement d'homogénéité. Le souci de faire apparaître plus clairement les faits d'importance générale l'a, d'autre part, emporté sur celui d'enrichir la carte en informations de détail : dans cet esprit, aucune source - ordinaire, minérale ou thermale — ni aucun captage, ne sont figurés.

\section{Mode de présentation}

Conçus à la fois de manière à faire apparaître le plus clairement possible la classification proposée, et ausi à permettre une édition rapide el économique, les modes de représentation choisis sont une création originale. La signification donnée aux figurés et couleurs s'est volontairement écartée, pour n'entrainer aucune confusion, des conventions appliquées pour les cartes géologiques ou les cartes hydrogéologiques.

Réalisation

La carte a été dressée rapidement novembre 1963 à janvier 1964) par le Bureau de recherches géologiques et minières, avec la collaboration pour la région de programme Languedoc, du Centre d'études et de recherches hydrogéologiques de l'Université de Montpellier. Elle est parue en novembre 1964.

Les promoteurs et les auteurs de la carte souha:tent que sa publication (1) suscite assez de critiques et incite assez de lecteurs à leur proposer des corrections, pour qu'une réédition, enrichie par des collaborations plus nombreuses, puisse être rapidement entreprise.

(1) La carte est diffusée par le Centre français d'information de l'eau (C.E.F.I.E.), 1, avenue Charles-Floquet, Paris$\left(7^{\circ}\right)$. 


\section{Discussion}

Président : M. BanaL

M. le Président remercie M. Margat de son exposé et souligne ce travail remarquable qui, ainsi que M. MARGa? l'a dit, a été fait en quelques mois, ce qui montre l'abondance des documents disponibles pour de telles synthèses.

En réponse à des questions de M. l'Inspecteur général René Lévy, de M. RÉménmóras et de M. Múnard, M. Margat confirme que la carte au $1 / 1000000^{\circ}$ qu'il a présentée n'existe qu'en France et précise que les cartes à même échelle existant dans d'autres pays, tels que l'Allemagne, sont des cartes hydrogéologiques, d'un niveau scientifique plus élevé. La Tunisie achève une carte hydrogéologique au $1 / 500000^{\circ}$. A ce sujet, il y a un certain retard en France.

Sur la demande de M. le président, M. Margat explique que les cartes hydrogéologiques sont, en général, à une échelle beaucoup plus grande $\left(1 / 50000^{\circ}\right)$. Il précise que les cartes locales, comme la carte de Douai, fournissent des indications quantitatives, moins sur l'aspect dynamique des eaux que sur leur position précise : surface piézométrique, situation des puits et des sources, débits des sources, transmissivités mesurées à la suite de forages, topographie souterraine des couches aquiferes, courbes de la base de la nappe, données hydrochimiques, informations sur les degrés d'exploitation actuelle, etc...

Les cartes hydrogéologiques sont beaucoup plus longues à établir (cinq à six ans pour la carte de Douai) que la carte présentée ici, qui donne une vue d'ensemble en attendant des cartes régionales plus précises.

D'autre part, les utilisateurs prévus ne sont pas tout i fait les mèmes : les cartes hydrogéologiques sont conçues, en partie, pour les hydrogéologues eux-mêmes; c'est un langage entre eux pour transmettre leurs connaissances, tandis que la carte présentée par M. Margat peut être comprise par toute personne capable de lire une carte en général.

M. MÉnard ayant remarqué que certaines nappes profondes ne sont pas représentées, M. Margat indique que l'on n'a pas cherché à se limiter par la profondeur, mais par l'état des connaissances ou la qualité de l'eau : par exemple, on a indiqué que la nappe des sables verts du bassin de Paris et celles des grès vosgiens ont leurs limites d'expansion propre, mais elles ont des limites de qualité, dues au fait qu'au-delà d'une certaine concentration ( $1 \mathrm{~g} / 1)$, l'eau est trop salée pour se prêter à certains usages.

M. Castany signale que le principe d'établissement d'une telle carte, qui a été mise au point pour la France, servira de base à une cartographie internationale au cours de la Décennie Hydrologique; il en est de même pour les cartes hydrogéologiques au $1 / 50000^{\circ}$. M. C CASTANY attire l'attention sur la collaboration très efficace de M. Margat dans ce domaine. 